\title{
Die tierärztliche Aufklärungspflicht
}

\author{
Kai Bemmann \\ Kanzlei Berner, Fischer \& Partner, Verden
}

\begin{abstract}
Vorwort
Die ärztliche Aufklärungspflicht ist eine Thematik, die in der Ärzteschaft diskutiert wurde, seitdem die Patientenschaft medizinkritischer wurde und der ärztliche Eingriff im Spannungsfeld mit den Grundrechten des Patienten gesehen wurde. Zwangsläufig befasste sich daraufhin auch die Rechtsprechung mit dieser Problematik. Sobald in der Humanmedizin Aufklärungs- oder andere Probleme juristisch diskutiert und von der Rechtsprechung behandelt werden, davert es nicht lange, bis die Tiermedizin überlegen muss, inwieweit auch sie von diesen Problemen berührt wird. Dies gilt insbesondere in der heutigen Zeit, weil die Patienten z. B. die Pferde oder Hunde immer wertvoller bzw. teurer werden. Derzeit ist die Aufklärungspflicht des Tierarztes besonders aktuell; denn die Gesellschaft für Pferdemedizin und die Bundestierärztekammer haben in Zusammenarbeit die Leitlinien zur Aufklärungspflicht in der Pferdepraxis erstellt. Damit hat die Tierärzteschaft einen wichtigen Schritt zur Eigenverantwortung unternommen. Bisher hat die Rechtsprechung durch Entscheidungen in Einzelfällen entscheidend die Maßstäbe bestimmt und die Tierärzteschaft musste reagieren, indem sie sich daran orientierte. Nun hat die Tierärzteschaft agiert und einen guten Weg gefunden, aus ihrer Sicht Maßstäbe zu setzen. Mit diesen wird sich die Rechtsprechung dann im Einzelfall auseinandersetzen müssen.
\end{abstract}

\section{Rechtliche Grundlagen der tiermedizinischen Aufklä- rungspflicht}

Die humanmedizinische Aufklärungspflicht und die dazu von der Rechtsprechung entwickelten Grundsätze über Art und Umfang der Aufklärung werden aus dem verfassungsrechtlich gesicherten Selbstbestimmungsrecht des Patienten abgeleitet. Dabei steht die Person des Patienten als Mensch im Vordergrund. Dessen Würde gilt es aus sittlichen Gründen zu achten (BGH 1959). All diese Gesichtspunkte spielen in der Tiermedizin keine Rolle. Selbst wenn gefühlsmäßige Bindungen zwischen Tierhalter und seinem Tier besonders stark ausgeprägt sein sollten, steht aus tiermedizinischer Sicht die wirtschaftliche Bedeutung des Tieres im Vordergrund. Diese Sichtweise wird durch die rechtlichen und sittlichen Gebote des Tierschutzes begrenzt (BGH 1977) Deshalb wird die tierärztliche Aufklärungspflicht auch nicht aus den Grundrechten abgeleitet, sondern als vertragliche Nebenpflicht des Behandlungsvertrages verstanden. Sie bestimmt sich im Einzelfall nach der Art der Behandlung des zu behandelnden Tieres und seinem materiellen und ideellen Wert sowie den Vorgaben des Auftraggebers (BGH 1980). Aufgrund der unterschiedlichen dogmatischen Ansätze für eine Aufklärungspflicht in der Humanmedizin und der Tiermedizin sind die für die Humanmedizin herausgearbeiteten Rechtsgrundsätze nur teilweise auf den tierärztlichen Behandlungsvertrag übertragbar. Für den Bereich der Aufklärungspflichten gilt dies aus den vorstehend genannten Gründen besonders. Die vereinzelt in der Rechtsprechung vertretene, gegenteilige Ansicht (OLG Oldenburg 1978) hat sich nicht durchgesetzt und ist vom BGH ausdrücklich abgelehnt worden (BGH 1980).

Der entscheidende Unterschied zwischen dem humanmedizinischen und dem tiermedizinischen Eingriff besteht darin, dass sich der humanmedizinische Eingriff nur vor dem Hintergrund der Einwilligung des Patienten überhaupt rechtfertigen lässt. Ohne Einwilligung ist er rechtswidrig und strafbar. Währenddessen reicht für den Tierarzt zur Rechtfertigung seines Eingriffs der seitens des Patienteneigentümers oder eines von ihm bevollmächtigten Vertreters erteilte Auftrag aus.

\section{Art und Umfang der tiermedizinischen Aufklärung}

Der Tierarzt ist seinem Auftraggeber gegenüber je nach Auftragsumfang verpflichtet, das Tier zu untersuchen und/oder zu behandeln. Dabei schuldet er den Einsatz der von einem gewissenhaften Veterinärmediziner zu erwartenden tiermedizinischen Kenntnisse und Erfahrungen (BGH 1980). Danach ist also der objektiv von einem praktischen Tierarzt zu erwartende Wissensstand - und nicht der des (ggfs. eine Klinik betreibenden) Fachtierarztes oder gar der eines in direktem Kontakt zur Wissenschaft stehenden Hochschullehrers - als allgemeiner Maßstab für die Art und den Umfang der geschuldeten Sorgfalt im Rahmen eines tierärztlichen Behandlungsund/oder Untersuchungsvertrages anzusehen. Dies gilt für alle Spektren des Vertrages, also auch für die Aufklärung.

Die Verpflichtung, überhaupt eine Aufklärung zu leisten, hängt für den Tierarzt in erster Linie davon ab, wie risikobehaftet sein Eingriff ist. Die sog. Komplikationsdichte der jeweiligen Behandlung und die wirtschaftliche Bedeutung, die eine Verwirklichung der Komplikation im jeweiligen Einzelfall erlangen könnte, sind die für den Tierarzt abzuwägenden Kriterien. Beide Kriterien gehen häufig ineinander über. Dies gilt insbesondere dann, wenn eine wenig wahrscheinliche Komplikation einen besonders hohen Wert gefährdet oder wenn ein Auftraggeber intensiv nachfragt oder ein geringes Misserfolgsrisiko im Falle seiner Verwirklichung zu einer Unbrauchbarkeit des Tieres führt. 
Die Kenntnisse und Erfahrungen der tiermedizinischen Wissenschaft bestimmen stets den Aufklärungsmaßstab (BGH 1983). Der Aufklärungsmaßstab muss deshalb mit der Fortentwicklung der Wissenschaft Schritt halten und dementsprechend ständig höheren Anforderungen genügen. Besteht allerdings ein wissenschaftlicher Meinungsstreit, könnte selbst dann keine schuldhafte Pflichtverletzung vorliegen, wenn objektiv Aufklärung geboten erscheint. Um solche Situationen zu vermeiden, ist der Aufklärungsleitfaden eine große Hilfe, weil er erheblich zur Sicherheit über den Stand der Wissenschaft in der Tiermedizin beiträgt. Sofern sich aus den Umständen des zu behandelnden Einzelfalles für den Tierarzł eine Aufklärungspflicht ergibt, verletzt er diese (nur) dann schuldhaft, wenn er aufgrund der Erkenntnisse von Schrifttum und Lehre auch davon ausgehen musste, dass eine Aufklärungspflicht besteht (OLG Celle 1989).

Allerdings muss die Tierärzteschaft aufpassen, mit ihrem Bestreben Leitlinien aufzustellen, nicht "Opfer" einer Leitlinienrechtsprechung zu werden, mit der sich die Humanmedizin seit einigen Jahren auseinanderzusetzen hat. Danach besteht die Gefahr, dass ein Abweichen von der Leitlinie bereits als grob fehlerhaft angesehen werden könnte (OLG Düsseldorf 2000) mit der Folge, dass der betroffene Tierarzł der Beweislastumkehr ausgesetzt wäre. Deshalb muss verdeutlicht werden, dass der Aufklärungsleitfaden den jeweiligen Stand der Wissenschaft widerspiegelt und dem Streben der Tierärzteschaft nach bestmöglicher Dienstleistungserbringung dienen soll, nicht aber den unbedingt einzuhaltenden Mindeststandard vorgeben soll.

\section{Darlegungs- und Beweislast}

Es gelten grundsätzlich die allgemeinen Regeln der Darlegungs- und Beweislast im Haftungsrecht. Danach hat der Anspruchsteller den Schaden, den Behandlungsfehler und auch den Ursachenzusammenhang zwischen beidem zu beweisen (BGH 1980; BGH 1989). Es genügt nicht, dass im Zusammenhang mit einer tierärztlichen Behandlung ein Schaden auftritt. Denn aufgrund der Eigendynamik, die ein in Behandlung befindliches Tier auch ohne Beteiligung eines Tierarztes entfalten kann, sieht die Rechtsprechung die Anwendung der Regeln über den Anscheinsbeweis, ebenso wie die Beweislastverteilung nach Gefahrenkreisen und auch die Anwendung von $\S 282$ BGB a. F. (bzw. jetzt $\S 280$ । S. 2 $B G B$ n. F.) als ausgeschlossen an (BGH 1977; BGH 1982). Nach der Beweislastregel des $\S 282$ BGB a. F., trägt der zur Leistung Verpflichtete die Beweislast dafür, dass eine ihm unmöglich gewordene Leistung ohne sein Verschulden unmöglich wurde. Für die nach der Schuldrechtsreform (01.01.2002) geschlossenen Vertragsverhältnisse ergibt sich eine gleiche Beweislastverteilung aus der Art der Gesetzesformulierung in $\S 280$ । S. 2 BGB n. F.

Von diesen Grundsätzen ist nur dann abzuweichen, wenn der Anspruchsteller dem Tierarzt einen groben Behandlungsfehler nachweist, der geeignet ist, den im Streit befindlichen Schaden auf nicht ganz unwahrscheinliche Weise herbeizuführen. In einem solchen Fall obliegt dem Tierarzt der Nachweis, dass der Schadenfall auch bei kunstgerechter Vorgehensweise eingetreten wäre (BGH 1981; BGH 1983). Insoweit ist die Rechtsprechung zur Haftung des Humanmediziners bei gro- ben Kunstfehlern auf die Haftung des Tiermediziners vollumfänglich anwendbar (OLG München 1990).

Als grob im rechtlichen Sinne ist nur ein solcher Fehler anzusehen, der dem Tierarzt bei objektiver Betrachtung generell nicht unterlaufen darf und unverständlich sowie unverantwortlich erscheint, weil ein Verstoß gegen bewährte tierärztliche Behandlungsregeln oder gesicherte medizinische Erkenntnisse vorliegt (BGH 1983). Allerdings ist grundsätzlich eine juristische Gesamtschau der Behandlung mit der Folge vorzunehmen, dass auch eine Mehrzahl "einfacher" Behandlungsfehler eine Behandlung im Lichte ihrer Gesamtbetrachtung als grob kunstfehlerhaft erscheinen lassen können (BGH 2001; OLG Köln 1991). Auch insoweit gelten die Regeln des Arzthaftungsrechts uneingeschränkt für die Tierarzthaftung.

Je klarer und eindeutiger die Maßstäbe sind, die sich die Tierärzteschaft selbst beispielsweise durch diese Leitlinien, durch die Beschlüsse der ersten und zweiten Röntgenkommission oder ähnliche Maßnahmen setzt, desto besser weiß der einzelne Tierarzt, welchen Standard er bei der Aufklärung zu erfüllen hat. Andererseits wird aber auch für jeden Tierarzt die Gefahr größer, bei einem Abweichen von den Leitlinien einen groben Kunstfehler zu begehen, wenn nämlich die Rechtsprechung die Leitlinie zum generellen Mindeststandard erhebt. Dem muss die Tierärzteschaft durch geeignete Formulierung aus den oben genannten Gründen im Ansatz entgegenwirken; denn die Leitlinie kann nur als wissenschaftlich begründete Entscheidungshilfe für die tiermedizinische Praxis dienen und nie die Einzelfallentscheidung ersetzen. Der Einzelfall kann vielmehr sogar eine von einer Leitlinie abweichende Vorgehensweise gebieten.

Im Unterschied zur Haftung des Humanmediziners muss im Tierarzthaftungsrecht allerdings der Anspruchsteller darlegen und beweisen, dass eine hinreichende Aufklärung nicht stattgefunden hat und diese Unterlassung ursächlich für den von ihm behaupteten Schaden geworden ist (BGH 1980). Die gegenteilige Ansicht des OLG Oldenburg aus dem Jahre 1978 lehnt der BGH ausdrücklich ab, weil die Aufklärung über und die Einwilligung in den Eingriff nicht wie in der Humanmedizin die Rechtmäßigkeitsvoraussetzung des Eingriffs darstellt. Das OLG Oldenburg hat von seiner Außenseitermeinung inzwischen ausdrücklich Abstand genommen und unter Hinweis auf die Rechtsprechung des BGH klargestellt, dass die humanmedizinischen Aufklärungsgrundsätze und -maßstäbe nicht für die Tiermedizin gelten (OLG Oldenburg 1998). Soweit auch heute noch in einigen instanzgerichtlichen Entscheidungen die Rechtsauffassung vertreten wird, dass in der Tiermedizin die humanmedizinischen Aufklärungsregeln anzuwenden sind, beruht dies regelmäßig auf Unkenntnis der anders lautenden BGH-Rechtsprechung. So hat beispielsweise auch noch das OLG Celle im Jahre 1997 behauptet, dass der Tierarzt genauso wie der Humanmediziner die Beweislast für die Erfüllung seiner Aufklärungspflicht trägt und dabei zur Begründung seiner Auffassung auf die Entscheidungen des BGH und des OLG Köln aus dem Jahre 1992 hingewiesen (OLG Celle 1997). Insoweit ist das Urteil allerdings offenkundig falsch. Dies erschließt sich bereits daraus, dass sich das OLG Celle mit dieser Entscheidung sowohl ohne Begründung in einen diametralen Widerspruch zu seiner bisherigen Rechtsprechung setzt, wonach die humanmedizinischen Aufklärungsgrundsätze in der Tiermedizin nicht 
anwendbar sind (OLG Celle 1989), als auch zur vermeintlichen Stütze der vertretenen Rechtsauffassung aus Entscheidungen des BGH und des OLG Köln zitiert, die sich überhaupt nicht mit der Haftung des Tierarztes befassen. Die in Bezug genommenen Entscheidungen ergingen zur Haftung des Humanmediziners im Rahmen einer Schilddrüsenoperation bzw. operativen Behandlung einer Stressinkontinenz. Diese Entscheidungen konnten dementsprechend gar keine Maßstäbe für die Haftung eines Tierarztes setzen.

Der $\mathrm{BGH}$ hat im übrigen mit seiner Entscheidung vom 19.01.1982 noch einmal ausdrücklich auch in Bezug auf die Aufklärungspflicht des Tierarztes klargestellt, dass die Rechtsgrundsätze zur Darlegungs- und Beweislast im Arzthaftungsrecht auf die Tierarzthaftung keine Anwendung finden und es bei der Darlegungs- und Beweislast des Anspruchsstellers bleiben muss, weil der Grund für die Verlagerung der Beweislast auf den Humanmediziner bei der Aufklärung über den humanmedizinischen Eingriff seine Ursache nur im Selbstbestimmungsrecht des Patienten hat, welches in der Tiermedizin keine Rolle spielt (BGH 1982). Ebenso wenig ist es gerechtfertigt, dem Tierarzt die Beweislast in Analogie zur Beweislastverteilung bei einem groben Behandlungsfehler aufzubürden (OLG Düsseldorf 1990), wenn ihm vom Anspruchsteller eine Aufklärungspflichtverletzung vorgeworfen wird.

\section{Beispiele aus der Rechtsprechung}

Behandlungsmethoden

Kommen mehrere Alternativen in Betracht, müssen diese Gegenstand der Erörterung sein (BGH 1980)

Die Anwendung der risikoreichen Alternative bedarf der besonderen Einwilligung des Patienteneigentümers (OLG Düsseldorf 1992)

\section{Dokumentation}

Aufklärungspflichtige Umstände unterliegen der tiermedizinischen Dokumentationspflicht (OLG Stuttgart 1996)

\section{Euthanasie}

Liegt keine notwendige Euthanasie vor, ist die Rechtswidrigkeit nur durch eine vorherige Einwilligung und Aufklärung des Tierhalters ausgeschlossen (OLG München 1991).

Ein Vertrag über die Einstellung eines Pferdes zum Zweck der tierärztlichen Behandlung in einer Klinik umfasst auch die Einwilligung in eine ggf. notwendige Tötung. Gleichwohl muss der Tierarzt den Tierhalter allerdings noch über die Entwicklung des Gesundheitszustandes und gegebenenfalls bestehende Behandlungsalternativen unterrichten (BGH 1982).

Die Tötung eines vom Jäger angeschossenen Hundes ist nur dann gerechtfertigt, wenn sie erforderlich ist, also nicht durch einen auf Verdacht abgegebenen Fangschuss (OLG Karlsruhe 1991).

\section{Gewebeentfernung}

Die Entfernung von Granulationsgewebe erfordert keine vorherige Aufklärung (OLG Hamburg 1989).

\section{Probelaparotomie}

Die Entnahme einer nicht notfallmäßig erforderlichen Probe-Laparotomie erfordert die Aufklärung über die Möglichkeit einer weniger belastenden Ultraschalluntersuchung (AG Stuttgart 1993).

\section{Honoraranspruch}

Versäumt es der Tierarzł schuldhaft, den Auftraggeber über die Behandlung aufzuklären, kann der tierärztliche Honoraranspruch entfallen, wenn der Auftraggeber infolge der unterlassenen Aufklärung die Behandlung durchführen lässt und der Behandlungserfolg ausbleibt (AG Großburgwedel 2002, das Gericht nimmt auf die Rechtsprechung zur ärztlichen Aufklärung [OLG Saarbrücken 2000] Bezug).

Ein tierärztlicher Honoraranspruch kann teilweise aus dem Gesichtspunkt einer pV bei nicht eindeutig indizierter Behandlung auch dann fortfallen, wenn ein Ursachenzusammenhang zwischen der Behandlung und einem Schaden am Pferd nicht besteht (LG Verden 2003) Anmerkung: Die zur Urteilsbegründung zitierte Literatur indiziert, dass sich das Gericht im Rechtsirrtum befand; denn diese Literatur vertritt eindeutig die entgegengesetzte Rechtsauffassung, wonach ein Honorarfortfall nur durch Aufrechnung mit Schadenersatzansprüchen erreichbar ist. In zweiter Instanz ist dem Tierarzt deshalb sein Honoraranspruch zuerkannt worden (OLG Celle 2004).

Der tierärztliche Honoraranspruch entfällt trotz Behandlungsfehler nur durch Aufrechnung mit Schadenersatzansprüchen aus pV, weil eine Schlechterfüllung der Dienstpflicht den vereinbarten Dienstlohn nicht berührt (AG Bad Segeberg 2003).

Impfungen und intramuskuläre Injektionen

Keine Aufklärungspflicht über mögliche Schockreaktionen (OLG Hamm 2000).

Injektionen anderer Art

Ist eine Schockreaktion bisher nicht bekannt geworden, bedarf die Injektion des Medikamentes keiner Aufklärung. Denn bei einem kleinen Eingriff ist über bisher nicht aufgetretene und nur theoretisch vorstellbare Risiken nicht aufzuklären (OLG Oldenburg 1998)

Der Tierarzt braucht vor einer i. v. Injektion nicht über die Gefahren einer Schockreaktion aufzuklären, wenn der Medikamentenbeipackzettel keine Hinweise auf eine solche Reaktion enthält; denn dann handelt es sich um ein nicht ernstlich in Betracht zu ziehendes Risiko (OLG Frankfurt 1991). 
Kastration

Beim 3jährigen und älteren Hengst ist die Kastration am stehenden Pferd eine auf ausdrücklichen Wunsch mancher Tierhalter angewendete, aber kunstfehlerhafte Methode, weshalb der Tierarzł hierfür das Einverständnis des Tierhalters ausdrücklich einholen muss (OLG Düsseldorf 1992).

Eine Kastration am stehenden Pferd kann bei einem älteren Hengst ausnahmsweise dann nicht schuldhaft kunstfehlerhaft sein, wenn andere Risiken hinzutreten, bei deren Berücksichtigung eine Entscheidung gegen die Kastration am abgelegten Pferd nicht schuldhaft falsch erscheint, sofern der Tierarzt die Einwilligung für die abweichende Methode einholt. Dies kann auch dadurch geschehen, dass der Tierarzt die Methoden und Risiken darstellt und der Tierhalter dem Tierarzt die Entscheidung überlässt. Hier: Seinerzeit besonders häufig nach Vollnarkosen auftretendes Thyphokolitisrisiko (LG Hannover 1997)

Nach anderer Ansicht ist die Kastration eines 3iährigen oder älteren Hengstes im Stehen nur dann gerechtfertigt, wenn der Tierarzł ausdrücklich auf die Abweichung von der tiermedizinischen Lehre und die Kunstfehlerhaftigkeit hinweist und eine solche Aufklärung auch noch beweist (OLG Celle 1997; kritische Anmerkung zur Beweislast unter Hinweis auf die Rechtsprechung des BGH siehe unter Kapitel: Darlegungs- und Beweislast).

Nach wiederum anderer Ansicht handelt es sich bei der Kastration am stehenden Pferd auch bei 3jährigen und älteren Hengsten um eine aus Kostengründen weit verbreitete Methode, so dass es ausreichend ist, auf die Alternative zu einer Operation im Liegen mit bedecktem Samenstrang hinzuweisen, die dann in einer Klinik durchzuführen sei. Das Abweichen von der Lehrmeinung ist nicht aufklärungspflichtig (LG Kassel 2002).

Isł die Entscheidung über die Durchführung der Kastration bereits getroffen, gilt die Entscheidungsfindung beim Auftraggeber als abgeschlossen und eine Aufklärung ist nicht mehr Gegenstand des erteilten Auftrages. Vielmehr schuldet der Tierarzt nun die aus tiermedizinischer und wirtschaftlicher Sicht günstigste Behandlungsmethode (OLG Frankfurt 2003).

Auch bei der Kastration eines 2jährigen Hengstes ist grundsätzlich über die verschiedenen Methoden und ihre Gefahren aufzuklären. Eine Aufklärung über die geringeren Risiken einer Kastration am abgelegten Pferd kann aber unterbleiben, wenn der Tiereigentümer sich bereits eingehend über die Kastrationsmethoden informiert zeigt und einen ambulant behandelnden praktischen Tierarzt beauftragt. Gleichwohl soll dann aber noch das Nachblutungsrisiko nach Durchführung des Eingriffs aufklärungspflichtig sein (OLG Zweibrükken 1996).

\section{Kehlkopfpfeifer-OP}

Der Tierarzt kann auch eine risikoreichere Operationsmethode ohne Aufklärung durchführen, wenn der Tierhalter schon anderweitig Aufklärung erfahren hat. Es besteht ohnehin keine Pflicht, unaufgefordert über alle Risiken einer tiermedizinischen Operation aufzuklären (OLG Karlsruhe 1982).
Kolik

Es ist ausreichend, dass der Tierarzt den Patienteneigentümer zum Zweck der selbständigen Nachsorge seines Pferdes darüber aufklärt, maßvoll zu füttern und zu schonen. Eine bestimmte Aufklärung über die Schonung im Einzelnen wie z. B. das Unterlassen eines Aufregung verursachenden Beschlages schuldet der Tierarzł nicht (OLG Saarbrücken 1990).

Ergeben sich bei einer ambulanten Klinikbehandlung Anzeichen eines Dünndarmverschlusses ist der Tierhalter sofort über die besseren Behandlungs- und Operationsmöglichkeiten, die bei stationärer Klinikbehandlung bestehen, aufzuklären (LG Detmold 1995).

\section{Kopper-OP}

Übergibt der Tierhalter sein Pferd dem Tierarzł mit dem konkreten Auftrag, eine Kopperoperation durchzuführen, schuldet der Tierarzł keine weitere Aufklärung über die Operationsrisiken (OLG Düsseldorf 1986).

\section{Narkose}

Keine Aufklärungspflicht über Vollnarkoserisiken (LG Köln 1992).

\section{Rektaluntersuchung}

Das Risiko der Darmperforation ist nicht aufklärungspflichtig (OLG Celle 1989).

\section{Schlussbemerkung}

Die Rechtsprechungsbeispiele sind Einzelfallentscheidungen, die in der Vergangenheit so getroffen wurden. Eine Rechtssicherheit, dass zukünftig in ähnlich gelagerten Fällen ebenso entschieden werden muss, kann daraus nicht abgeleitet werden. Denn einerseits herrscht richterliche Unabhängigkeit. Ein anderes Gericht, welches möglicherweise auch noch durch einen anderen Sachverständigen abweichend beraten wird, kann also denktheoretischerweise einen gleich gelagerten Haftungsfall anders entscheiden. Andererseits gelten die unter Kapitel 3 bereits erläuterten, von der Rechtsprechung erarbeiteten allgemeinen Aufklärungsgrundsätze. Sofern sich also der Stand der tiermedizinischen Wissenschaft ändert und in Schrifttum und Lehre darüber berichtet wird, erhöht sich der Aufklärungsmaßstab entsprechend.

Letztendlich muss der behandelnde Tierarzt im Einzelfall abwägen und entscheiden, ob er sogar überobligatorisch aufklärt und dabei den Zeitaufwand und die Hemmung im Praxisablauf im Kauf nimmt oder ob eine überobligatorisch umfangreiche Aufklärung den Patienteneigentümer nur verunsichert und behindert, die Entscheidung über den notwendigen Behandlungsauftrag zu treffen. Der Tierarzt muss deshalb zwangsläufig der Aufklärungspflicht in der Notfallbehandlung anders als im Rahmen einer nicht notwendigen aber vertretbaren Behandlung nachkommen. 


\section{Abkürzungen}

a.F

$A G$

$B G B$

$\mathrm{BGH}$

LG

n.F.

NJW

NJW-RR

NFWE-VHR

OLG

OLGR

$\mathrm{R}+\mathrm{S}$

VersR alte Fassung des BGB bis 31.12.2001

Amtsgericht

Bürgerliches Gesetzbuch

Bundesgerichtshof

Landgericht

Neue Fassung des BGB ab 1.1.2002

Neve juristische Wochenschrift

Neve juristische Wochenschrift - Rechtspre chungsreport Zivilrecht

Neue juristsiche Wochenschrift - Entschei dungsdienst Versicherungs- und haftungs recht

Oberlandesgericht

OLG Report, Schnelldienst zur Zivilrecht sprechung der Oberlandesgerichte Recht und Schaden (Zeitschrift)

Versicherungsrecht, Haftungs- und

Schadensrecht (Zeitschrift)

\section{Literatur}

AG Bad Segeberg (2003): Urt. v. 30.10.2003 Az: 17a C 263/99 AG Großburgwedel (2002): Unt. v. 18.09.2002, Az: 70 C 250/01 AG Stuttgart (1993): Urt. v. 26.08.1993, Az: 9 C 9443/92

BGH (1977): Der prakt. Tierarzt, $754 \mathrm{ff}$.

BGH (1959): NJW, 811

BGH (1977): NJW, 1102

BGH (1980): NJW, 1333

BGH (1980): NJW, 1904

BGH (1981): NJW, $2513 \mathrm{f}$

BGH (1982): NJW, 1327 f

BGH (1983): NJW, $333 \mathrm{ff}$.

BGH (1983): NJW, $2078 \mathrm{f}$

BGH (1983): NJW, $2080 \mathrm{ff}$.

BGH (1989): NJW, 554

BGH (1992): NJW, 2351

BGH (2001): NJW, $2792 \mathrm{ff}$

LG Detmold (1995): Urt. v. 21.06.1995 Az: 9 O 483/93

LG Hannover (1997): Urt. v. 30.01.1997 Az: 19 ○ 24/95

LG Kassel (2002): Urt. v. 22.08.2002 Az: 9 ○ 33/01

LG Verden (2003): Urt. v. 25.09.2003 Az: 5 O 448/99

LG Köln (1992): VersR, 207

OLG Oldenburg (1978): NJW, $594 \mathrm{f}$

OLG Karlsruhe (1991): NJW, $116 \mathrm{f.}$

OLG Celle (1989): NJW-RR, $539 \mathrm{ff}$.

OLG Frankfurt (1991): NJW-RR, $476 \mathrm{f}$

OLG Köln (1991): NJW-RR, 800 ff.

OLG Köln (1992): NJW-RR, 986

OLG München (1991): NJW-RR, $477 \mathrm{f}$

OLG Zweibrücken (1996): NJWE-VHR, $147 \mathrm{ff}$.

OLG Celle (1997): Urt. v. 22.10.1997, Az: 21 U 13/97

OLG Celle (2004): Urt. v. 23.04. 2004, Az: 21 U 59/03

OLG Frankfurt (2003): Urt. v. 31.10. 2003, Az: 15 U 65/03

OLG Hamm (2000): OLGR, 173

OLG Saarbrücken (2000): OLGR, 401

OLG Saarbrücken (1990): R+S, $300 \mathrm{f}$.

OLG Celle (1989): VersR, $640 \mathrm{ff}$.

OLG Düsseldorf (1986): VersR, $61 \mathrm{ff}$.

OLG Düsseldorf (1990): VersR, $867 \mathrm{ff}$.

OLG Düsseldorf (1992): VersR, $206 \mathrm{f}$.

OLG Düsseldorf (2000): VersR, $1019 \mathrm{ff}$.

OLG Hamburg (1989): VersR, 1105

OLG Karlsruhe (1982): VersR, $707 \mathrm{f}$.

OLG München (1990): Deutsches Tierärzteblatt 38, 84

OLG Oldenburg (1998): VersR, $902 \mathrm{f}$.

OLG Stuttgart (1996): VersR, $1029 \mathrm{f}$.

OLG Zweibrücken (1996): VersR, 1030 f.

Kai Bemmann

Rechtsanwalt und öffentlich bestellter und vereidigter

Sachverständiger für Pferdezucht und -haltung

Kanzlei Berner, Fischer \& Partner

Andreaswall 2

27283 Verden

bemmann@bernerfischer-partner.de

\section{Mit Abstand die meisten juristischen Streitigkeiten in der Pferdepraxis betreffen die Kaufuntersuchung!}

\section{Mit Abstand den besten Schutz bietet der Pferdeheilkunde-Vertrag über die Untersuchung eines Pferdes - Das Original!}

